Document downloaded from:

http://hdl.handle.net/10251/181093

This paper must be cited as:

Parra-Boronat, M.; Parra-Boronat, L.; Lloret, J.; Mauri, PV.; Llinares Palacios, JV. (2019). Low-cost Soil Moisture Sensors Based on Inductive Coils Tested on Different Sorts of Soils. IEEE. 616-622. https://doi.org/10.1109/IOTSMS48152.2019.8939258

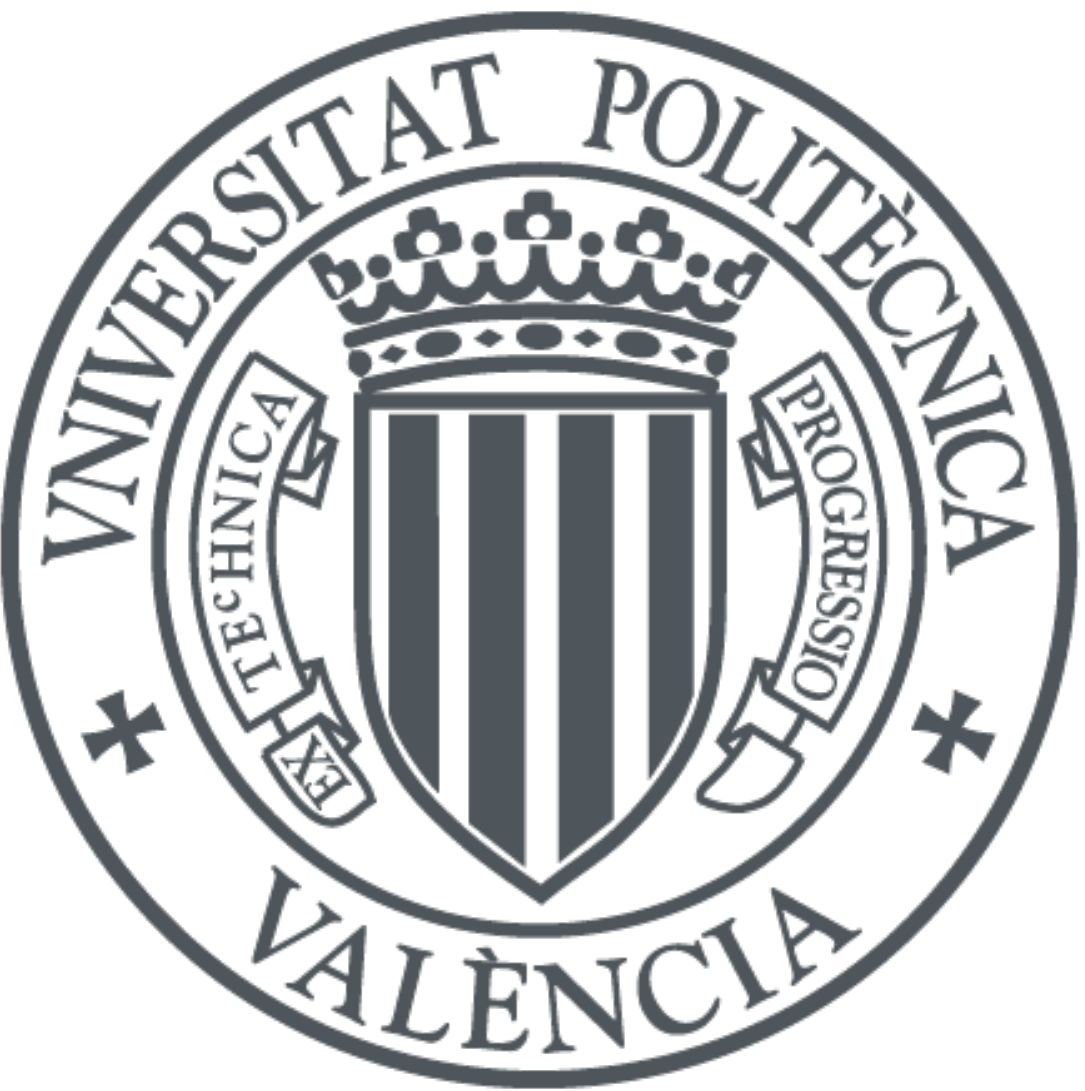

The final publication is available at

https://doi.org/10.1109/IOTSMS48152.2019.8939258

Copyright IEEE

Additional Information 


\title{
Low-cost Soil Moisture Sensors Based on Inductive Coils Tested on Different Sorts of Soils
}

\author{
Mar Parra ${ }^{1}$, Lorena Parra ${ }^{1,2}$, Jaime Lloret ${ }^{1}$, Pedro V. Mauri ${ }^{2}$ and Josep V. Llinares ${ }^{3}$ \\ ${ }^{1}$ Instituto de Investigación para la Gestión Integrada de Zonas Costeras \\ Universitat Politècnica de València, Valencia, Spain \\ ${ }^{2}$ Instituto Madrileño de Investigación y Desarrollo Rural, Agrario y Alimentario (IMIDRA), Finca "El Encin”, A-2, Km 38, \\ 2, 28800 Alcalá de Henares, Madrid, Spain \\ ${ }^{3}$ Mediterranean Agroforestry Institute, Universitat Politècnica de València, Camino de Vera s/n, 46022 Valencia, Spain \\ maparbo@epsg.upv.es, loparbo@doctor.upv.es, jlloret@dcom.upv.es, pedro.mauri@madrid.org, jollipa@qim.upv.es
}

\begin{abstract}
The use of precision agriculture and the Internet of Things has improved the efficiency of many cultures. Nevertheless, there are a few low-cost options to monitor soil moisture. Moreover, those options depend on the specific characteristics of the soil. In this paper, we attempt to find a sensor, based on mutual inductance, that could be used for more than one sort of soil. We study three prototypes, one of them with casing. The sensors are powered with a voltage of 10 peak to peak volts. One of the soils has a high content of organic matter and sand while the other is rich in sand and silt. The best prototype for the soil with high levels of organic matter has 10 turns on the powered coil and 5 on the induced coil. The best frequency for this sensor is $1340 \mathrm{kHz}$. For the soil with a significant quantity of silt, the best prototype has 80 turns on the powered coil and 40 on the induced coil. The frequency at which this sensor works best is $229 \mathrm{kHz}$, which happens to be its peak frequency. With those characteristics regressions lines with $\mathbf{R}^{2}$ values higher than 0.75 can be modeled.
\end{abstract}

Keywords-precision agriculture, IoT, mutual inductance, solenoid, conductivity sensor, water management

\section{INTRODUCTION}

Agriculture is the principal economic activity in most of the rural areas of the world. In most parts of the word it is the economic activity with the highest water requirements. Regarding the water scarcity and the exacerbation with climate change, the regulation of water usage is a crucial issue. The precision agriculture proposes to use sensors and remote sensing (both with satellite and drones) to monitor the performance of agriculture with the aim of adjusting the inputs (water, fertilizers, nutrients, etc). Several papers have presented different systems for precision agriculture.

Internet of things (IoT) has become a hot topic in the last years. The interconnection of different devices to share information and send data have multiple applications. It has been applied in many different fields, since industry [1], or surveillance [2] to e-health [3] or smart cities [4].

IoT can be also applied in conjunction with precision agriculture to reach the sustainability of the activity. The main drawbacks that are deterring the adoption of precision agriculture are: (i) the cost of sensors; (ii) the lack of specialized systems for different farming systems; and (iii) the little trust of the farmers on these systems.

If we pretend to boost the adoption of these systems, we need to offer low-cost systems, which have been tested in different farming systems and are robust and reliable. This will enhance the approval by the farmers. Currently, many systems have been proposed for monitoring agriculture. Generally, the proposed systems are composed of sensors which measure different physical variables, a smart algorithm that according to the measures triggers different actuators [5]. Most of the proposals are presented for a specific farming system as greenhouses and grain fields. Most of the systems for greenhouses include the aforementioned sensors and actuators. On the other hand, in grain fields is more common to find systems based on remote sensing to monitor the performance of crops and the actuators are located in the machinery. When sensors are to be used in the field, they must be properly designed and tested under different scenarios. One of the most used sensors in precision agriculture is the soil moisture sensors. However, the current low-cost soil moisture sensors are based on electric conductivity. Those sensors have two downsides (i) the sensing element (electrodes) must be in contact with the soil; and (ii) some sorts of soil might have salts and given the same water content the measure of conductivity can be different. The use of inductive soil moisture sensors was reported in [6], nonetheless the authors only test their prototypes with one sort of soil. Therefore, more tests are needed to ensure the suitability of this soil moisture sensor for precision agriculture.

In this paper, we present the comparison of two low-cost soil moisture sensor based on electromagnetic fields tested with different sort of soils. The first soil is a substrate commonly used in gardening, with a high content of organic matter and nutrients and a high percentage of sand. The second soil is a fallow land with a low percentage of organic matter, low nutrients, a significant quantity of silt (about 30\%) and a high percentage (about a $60 \%$ ) of sand. Three prototypes of the moisture sensor were tested. The different prototypes were previously tested to ensure that they are able to measure the soil moisture. The used prototypes are the ones that offered better results in previous tests. In this experiment, we use a different method to generate soil moistures, two sorts of soil, and a wider range of moistures.

The remainder of the paper is structured as follows, Section 2 presents the related work. Section 3 presents the material and methods used in the experiments. The results are presented in Section 4. Finally, Section 5 sets out the conclusions of this contribution.

\section{RELATED WORK}

In this section, we summarize the related work and identify the gap in the current solutions for soil moisture monitoring.

According to the review of Susha Lekshmi et al. in 2014 [7], there are 13 different methods to measure the soil moisture (including optical, dielectric and thermal techniques among others). However, not all these techniques can be implemented 
in sensors. The dielectric techniques are reported as quite reliable. Nonetheless, the given commercial sensors are expensive and regarding the capacitance probes, their deployment might be tricky. Kizito et al. in 2008 [8] compare different low-cost soil moisture sensors based on capacitive effect and concludes that the temperature affects to the measures. They found that the best measurement frequency is $70 \mathrm{MHz}$, at this frequency a single calibration curve can be used for all the tested sorts of soil. Though, the measuring frequency is too high. However, in [6] other sensors are presented with lower measuring frequencies. The measuring frequency is directly related to energy consumption. If we pretend to have sensors deployed for long-term we need to reduce their energy consumption, and decrease the measuring frequency is one option.

Many authors have claimed the importance of using soil moisture sensors in precision agriculture systems in many different crops. Gendrona et al. in 2018 [9] showed the benefits in water use and productivity of monitoring real-time irrigation in strawberries. They measure the soil matric potential as an indicator of soil moisture. They presented a decision-making tool for growers with regard to the adoption of irrigation techniques. The use of soil moisture sensor for monitoring drip irrigation in dwarf cherry trees was proposed by Dursun and Ozden in 2011 [10]. They suggest the use of a commercial soil moisture sensor (from Decagon) and a series of valves and pumps for irrigation. They showed the data gathered by the commercial sensor during 2:30 hours and the volumetric soil moisture changed from 16 to $19\left(\mathrm{~m}^{3} / \mathrm{m}^{3}\right)$. The utilized sensors were low-cost sensors based on two electrodes. The sensors were not calibrated according to the characteristics of the soil. The utilized sensor must be in contact with the soil and the authors do not report the performance of this sensor in the long term.

Mittelbach et al. in 2012 [11] set out the comparison of four low-cost commercial soil moisture sensors during 1 year. They compare the 10HS, CS616, TRIME and SISOMOP sensors. They establish that none of the tested sensors accomplishes the level of performance specified by the manufacturers. They point out that a specific coil calibration will need to improve the accuracy of the sensors. Furthermore, they affirm that some of them have a serious lack of sensitivity and a suspicious dependence on soil temperature. Therefore, low-cost commercial sensors must be improved before boosting the adoption of those sensors in precision agriculture.

Soulis and Elmaloglou in 2018 [12] stated the importance of sensors location for soil moisture monitoring in the case of layered soils. They proposed the combination of different sorts of sensors, with different sensibilities, in one probe. Each sensor was located at a certain depth. Thus, in one probe they can measure the soil moisture at different depth. Up to 6 probes were deployed jointly to obtain a map of soil moisture in the vicinity of the roots.

We can conclude that to have an optimal soil moisture sensor for an IoT precision agriculture system it must accomplish the following requirements: Low-cost, lowenergy consumption, long-term measurement capacity, calibrated with different sorts of soils. Nowadays, none of the available sensors can accomplish these requirements. In this paper, we present a sensor that accomplishes all these requirements.

\section{MATERIAL AND METHODS}

In this section we will explain the procedure through which the experiment was conducted. Moreover, we will list all the materials needed for the process. In order to better understand this, we will divide this section into two subsections. The first one will explain how the samples will be prepared. The second one will deal with the measures with the sensors.

\section{A. Coils description}

The sensor uses the phenomenon of mutual inductance [6]. It has two coils, one of which is powered (PC) and another one which is induced (IC). When the PC is powered with an electric current (EC) it generates a magnetic field. If the IC is in the proximity of the magnetic field it is affected by it and generates a magnetic flux. The voltage output (Vout) of the $\mathrm{PC}$ depends on the magnetic field generated by the PC and the medium through which it moves. The magnetic field and the induced voltage also depend on the number of spires (NS), the diameter of wire $(\varnothing \mathrm{W})$ and the diameter of the coil on each coil (ØРC and ØIC).

For this experiment, we used three prototypes.. The three of them present the same $\varnothing \mathrm{W}$, which is $0.4 \mathrm{~mm}$. . The characteristics of P1 to P3 can be seen in Table I. Moreover $\mathrm{P} 1$ presents casing, neither $\mathrm{P} 2$ nor $\mathrm{P} 3$ are endowed with one, see Fig. 1 and 2.

\section{B. Soil collection and preparation}

In order to test these prototypes on different soils, we will collect two samples. The first sample (S1) is composed of substrate for plants, rich in organic matter. The second sample (S2) will come from a field previously used to harvest citrus, it will be rich in silt and clay. The two samples will be prepared using the same protocol. First, the soil will be collected from its source. S1 will be bought at a gardening store. Meanwhile, S2 will be dug from a culture. For this step we will need a shovel and a recipient to put the soil in, like a bucket. It will be subtracted with care to do not grab rocks and plants. Second, the soil will be laid on a tray and with the aid of a rolling pin, we will break the possible clods.

\section{TABLE I. PROTOTYPES CHARACTERISTICS}

\begin{tabular}{|c|c|c|c|}
\cline { 2 - 4 } \multicolumn{1}{c|}{} & P1 & P2 & P3 \\
\hline Diameter of Inner PVC tube (mm) & 25 & 25 & 25 \\
\hline Layers & 8 & 1 & 1 \\
\hline Casing & Yes & No & No \\
\hline Separation between coils (mm) & 10 & 5 & 5 \\
\hline Diameter of Casing PVC tube (mm) & 43 & & \\
\hline Number of Windings of PC & 80 & 5 & 10 \\
\hline Number of Windings of IC & 40 & 10 & 5 \\
\hline
\end{tabular}

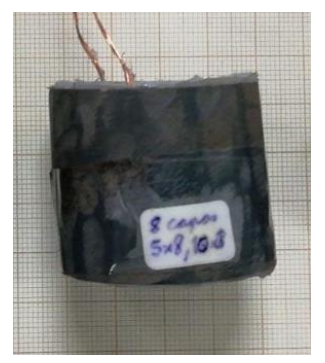

Fig. 1. P1 over milimitrated paper

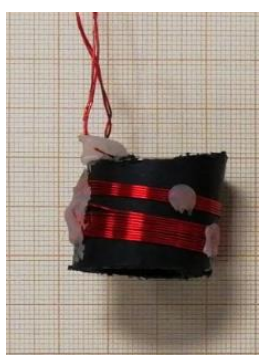

Fig. 2. P2 and P3 over milimitrated paper 
In this step, we will also remove any possible rock, plant or invertebrate that could be present. Then, we will use a $2 \mathrm{~mm}$ aperture sieve to filter the soil. We will do it in order to make sure that we are only testing the soil. We will repeat this procedure until we have at least kilo and a half of soil.

After this, we will prepare the pots. Before starting the procedure, we will take the necessary measures. The pots which will be used in this experiment are conical trunks. Therefore, the measures needed to calculate the volume using the conic trunk formula (Eq. 1) are the minor radius, the major radius and the height. The major radius and the height can only be measured once the soil is on the pot, but the minor radius has to be measured now.

$$
V=\frac{1}{3} \cdot \pi \cdot l \cdot\left(R^{2}+r^{2}+R \cdot r\right)
$$

\section{Moisturizing soils}

First of all, the bottom of the pot will be sealed with filter paper. This way, water can get in and out, but the soil will remain inside. The pot, along with the filter paper, will be weighed. Following, pots will be filled with $500 \mathrm{~g}$ of soil. The pot will then be put inside a plastic box or any other sort of container. The box will be filled with water up to one centimeter under the soil level inside the pot. We will wait until the upper layer of soil looks wet. Then, we will add another $500 \mathrm{~g}$ of soil and raise the level of water on the outside to one centimeter below the soil level. This process will be repeated a third time until the soil is completely wet. This process makes sure that the entirety of the soil is wet. In order to get rid of the excess of water, the pot will be left at 25 Celsius degrees for 24 hours.

Meanwhile, we will weight a small portion of the filtered soil. This portion will be heated to 100 Celsius degrees in order to evaporate all the water. After the evaporation it will be weighed again and the percentage of dry soil will be determined with the adequate formula (Eq. 2).

$$
\text { Dry soil } \%=\frac{\text { Weight of dry soil }(g)}{\text { Initial weight of soil }(g)} \cdot 100
$$

After the aforementioned 24 hours, the pot will be weighed. First we will subtract the weight of the pot and filter paper. Secondly, we will multiply by the percentage of dry soil. The result should be the weight of the dry soil. We will use this weight to calculate the soil moisture each day. This will be done with both soil samples. The soil samples can be seen in Fig. 3, the different characteristics of soil (colour, content of organic matter, and texture).

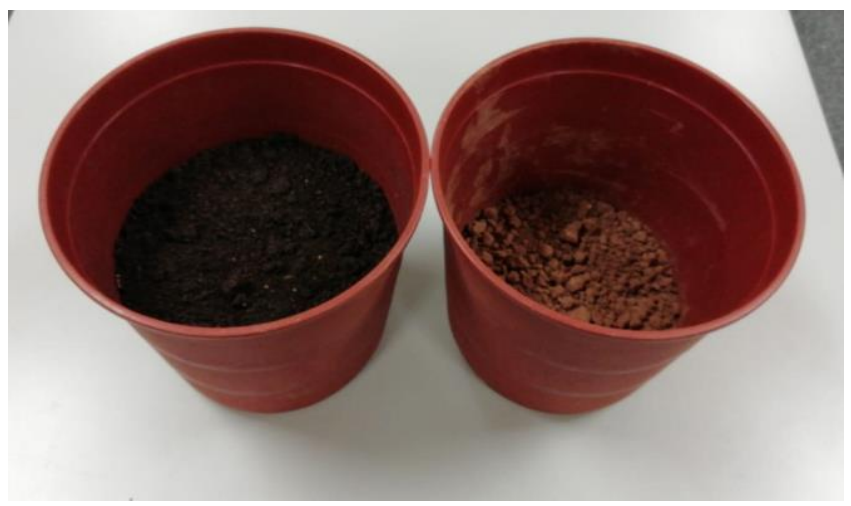

Fig. 3. The pots willed with the soil samples

Moreover, we will measure the major radius and the height for each sample. With this data we will be able to calculate the soil volume using the aforementioned formula for a conic trunk (1).

\section{Measuring procedure}

We will measure for six weeks, a total of six times, over the course of one month. The PC of the sensor will be powered with a current generator AFG1022 from Tektronix [13] that generates a 10 peak to peak voltage sinus-wave. The Vout will be measured with the oscilloscope TBS1104 from Tektronix [14]. Moreover, the PC will require a resistance of $47 \Omega$ connected to the positive wire. Furthermore, the IC will need a capacitor of $10 \mathrm{nF}$ connected to both wires. The complete set up should have the pot, the sensor, the current generator, the oscilloscope, the resistance and the capacitor, see Fig. 4 and 5.

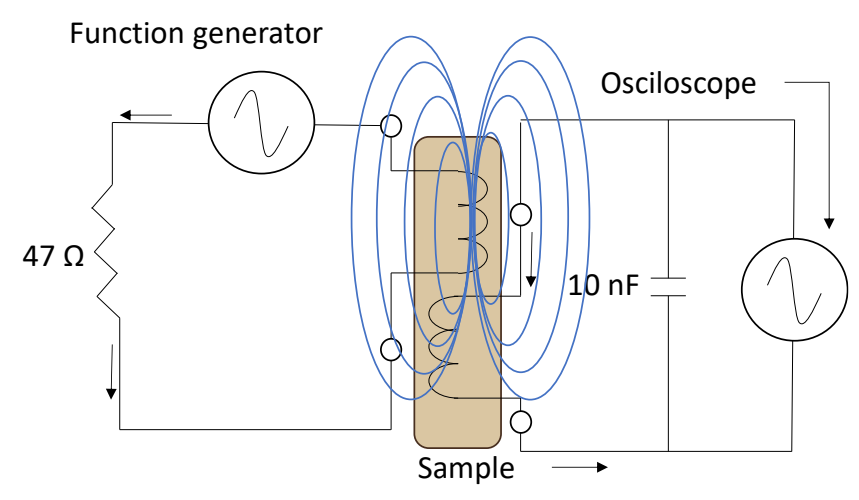

Fig. 4. Scheme of the circuit

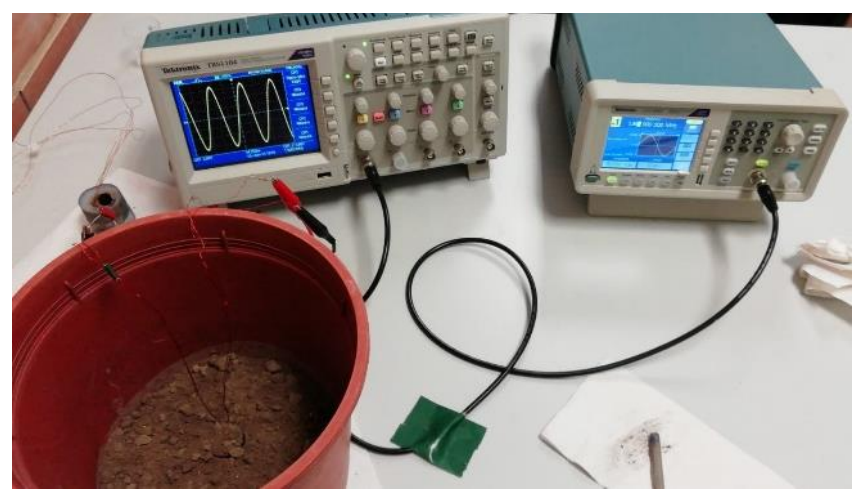

Fig. 5. Photography of the circuit

We will gather the data over the course of six weeks, measuring once per week. The three prototypes will be used with S1 and S2. To gather the data we will weigh the pots before measuring. We will subtract the weight of the dry soil along the weight of the pot and the filter paper. With this, we will obtain the weight of the water. Since the density of water is $1 \mathrm{ml} / \mathrm{g}$ we will be able to know the water volume. This value will be divided by the soil volume in order to obtain the soil moisture (Eq. 3).

$$
\text { Soil moisture }(\% \text { vol })=\frac{\text { Volume water }(L)}{\text { Volume soil }(L)}
$$

The PC will be powered with different EC around the peak frequency of each prototype, as well as the peak frequency. The different $\mathrm{EC}$ will derive $5 \mathrm{kHz}$ from one another and be multiples of five, the peak frequency being the only outlier. The process through which the soil moisture was estimated is shown. The substrate used for S1 is very rich in organic matter, therefore a high-water content is to be expected. We weigh $20 \mathrm{~g}$ of soil on a $3 \mathrm{~g}$ capsule, obtaining a total weight of $23 \mathrm{~g}$. After drying it, the total weight of the capsule Is $10 \mathrm{~g}$. 
Consequently, the dry soil weight is $7 \mathrm{~g}$. For S2, $25 \mathrm{~g}$ of soil are weighed on the capsule, the same used for S1. After being completely dried, the weight of the soil and the capsule is 24 g. Therefore, the dry soil weighs $21 \mathrm{~g}$. The results for both soils, after applying the proper equation (2) and performing the needed subtractions and additions are shown in Table II.

TABLE II. SOIL WEIGHT CHARACTERISTICS

\begin{tabular}{|c|c|c|}
\hline Characteristics & S1 & S2 \\
\hline Initial soil weight $(\mathrm{g})$ & 20 & 25 \\
\hline Capsule weight $(\mathrm{g})$ & 3 & 3 \\
\hline Dry soil weight $(\mathrm{g})$ & 7 & 21 \\
\hline Dry soil percentage & 35 & 84 \\
\hline Total soil weight $(\mathrm{g})$ & 1500 & 1500 \\
\hline Total dry soil weight $(\mathrm{g})$ & 525 & 1260 \\
\hline Pot and filter paper weight $(\mathrm{g})$ & 149 & 149 \\
\hline Dry soil, pot and filter paper weight $(\mathrm{g})$ & 674 & 1409 \\
\hline
\end{tabular}

The volume is calculated after taking the appropriate measures (height and major radius) for each pot. The minor radius is measured before adding the soil. It is to be noted that even though both pots are conical trunks they are slightly different. The pot for $\mathrm{S} 2$ is wider at the top than the pot for S1. The volumes for each are shown in Table III.

TABLE III. SOIL VOLUME

\begin{tabular}{|c|c|c|}
\hline Characteristics & S1 & S2 \\
\hline Minor radius $(\mathrm{cm})$ & 8 & 8 \\
\hline Major radius $(\mathrm{cm})$ & 8.5 & 8.75 \\
\hline Height $(\mathrm{cm})$ & 10.5 & 5 \\
\hline Volume $(\mathrm{cm} 3)$ & 2246 & 1103 \\
\hline
\end{tabular}

The initial water weight of S1 is $2363 \mathrm{~g}$. In the case of S2, the initial weight was $1888 \mathrm{~g}$. Using the pertinent formula (3) we can obtain their water volume. Said water volume is $75.21 \%$ for $\mathrm{S} 1$ and $43.45 \%$ for $\mathrm{S} 2$.

\section{RESULTS}

In this section, the results from the experiments previously explained are shown. In order to better explain them, this section is divided into three subsections. The first one presents the estimation of the water content on the pots. The graphs with the Vout for each sensor and pot combination are shown in the second subsection. In the third subsection, whether the same sensor can be used for both soils with the same equation or not is studied.

\section{A. Water content estimation}

In this subsection, the results for the soil moisture measures are presented. Not only in percentage but also in volume and weigh. These measures are key for the calibration of the prototypes. The initial water weight of S1 is $2363 \mathrm{~g}$ and after two weeks it weighs $2181 \mathrm{~g}$. Considering the volume of soil, the percentages of water (soil moisture) are shown in Table V. In the case of S2, the initial weight was $1888 \mathrm{~g}$. The results for the soil moisture are shown in Table VI. These results are used to compare the Vout of the sensors.
In this subsection, the results from the tests performed with the prototypes are shown. The Vout from the IC was measured every $5 \mathrm{kHz}$ around the peak frequency and on the peak frequency itself.

TABLE IV. S1 SOIL MOISTURE PERCENTAGE

\begin{tabular}{|c|c|c|c|}
\hline $\begin{array}{c}\text { Total } \\
\text { weight } \\
(\mathbf{g})\end{array}$ & $\begin{array}{c}\text { Water } \\
\text { weight } \\
\text { (g) }\end{array}$ & $\begin{array}{c}\text { Water } \\
\text { volume } \\
(\mathbf{c m 3})\end{array}$ & $\begin{array}{c}\text { Water } \\
\text { percentage } \\
\text { \% }\end{array}$ \\
\hline 2363 & 1689 & 1689 & 75.21 \\
\hline 2277 & 1603 & 1603 & 71.38 \\
\hline 2181 & 1507 & 1507 & 67.10 \\
\hline 2086 & 1412 & 1412 & 62.87 \\
\hline 1982 & 1308 & 1308 & 58.24 \\
\hline 1833 & 1159 & 1159 & 51.61 \\
\hline
\end{tabular}

TABLE V. S2 SOIL MOISTURE PERCENTAGE

\begin{tabular}{|c|c|c|c|}
\hline $\begin{array}{c}\text { Total } \\
\text { weight } \\
(\mathbf{g})\end{array}$ & $\begin{array}{c}\text { Water } \\
\text { weight } \\
(\mathbf{g})\end{array}$ & $\begin{array}{c}\text { Water } \\
\text { volume } \\
(\mathbf{c m 3})\end{array}$ & $\begin{array}{c}\text { Water } \\
\text { percentage } \\
\text { \% }\end{array}$ \\
\hline 1888 & 479 & 479 & 43.45 \\
\hline 1840 & 431 & 431 & 39.09 \\
\hline 1762 & 353 & 353 & 32.02 \\
\hline 1743 & 334 & 334 & 30.29 \\
\hline 1598 & 189 & 189 & 17.14 \\
\hline 1573 & 164 & 164 & 14.88 \\
\hline
\end{tabular}

The results from the P1 on S1 show a peak Vout of $31.2 \mathrm{~V}$ on several levels of soil moisture. This was obtained on the peak frequency, $229 \mathrm{kHz}$. It is possible that this value is a threshold, thus explaining its behavior. The measures do not seem to show a pattern, as seen in Fig. 6. Therefore, this prototype is not useful for $\mathrm{S} 1$.

The data from $\mathrm{P} 1$ on $\mathrm{S} 2$ show an increasing trend for most tested frequencies. The peak Vout observed with this prototype is of $30.8 \mathrm{~V}$, on the peak frequency as well. This prototype shows big differences between two contiguous frequencies, which makes the changes between different water percentages look small. The frequency $225 \mathrm{kHz}$ seems to show an uninterrupted increasing trend, as seen in Fig. 7. This frequency should be further studied.

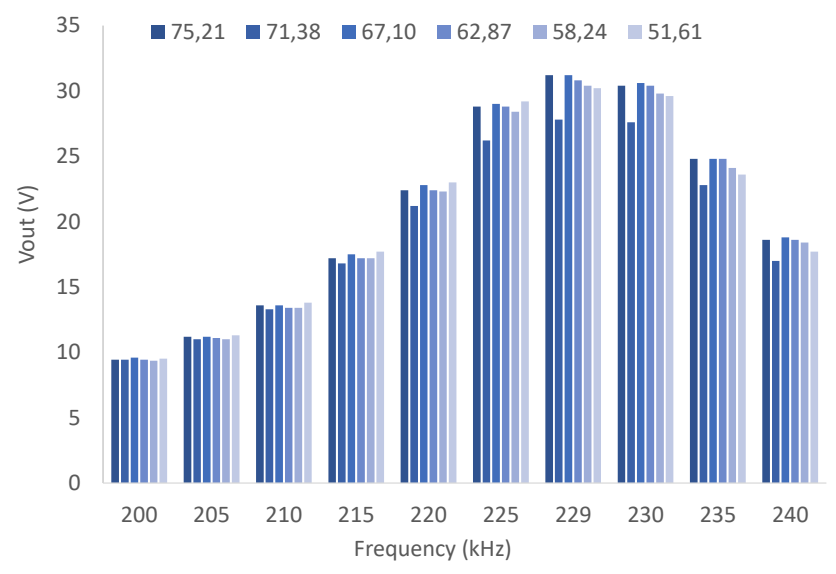

Fig. 6. Vout of P1 using S1

\section{B. Study of the Vout}




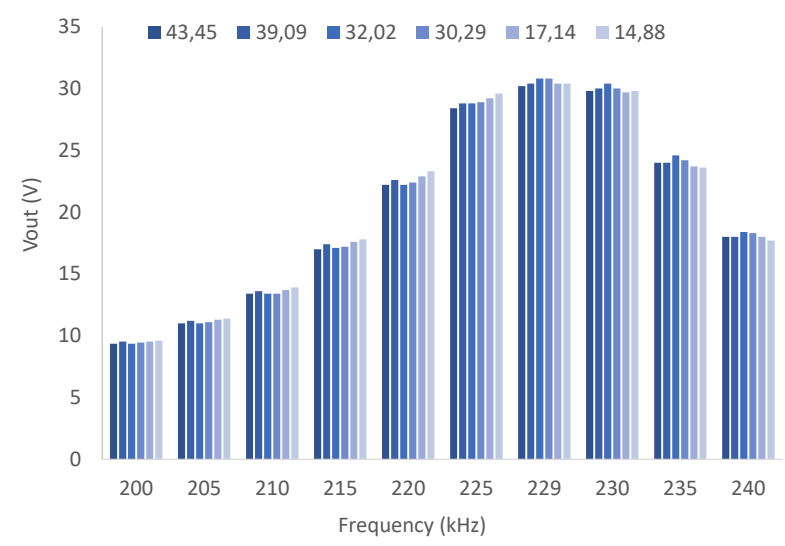

Fig. 7. Vout of P1 using S2

The second prototype, $\mathrm{P} 2$, shows an increasing trend up to the peak frequency when tested on S1. Beyond that frequency, they seem to have no correlation at all. The peak Vout is 14.6 $\mathrm{V}$, observed at $775 \mathrm{kHz}$ with almost $60 \%$ of water content. This prototype shows bigger differences than P1. Moreover, it seems to follow a step behavior, as seen in Fig. 8. The differences in $760 \mathrm{kHz}$ should be studied.

The same prototype when tested on S2 showed significantly worse results. The Vout seems to increase and decrease arbitrarily. The peak Vout, $14.7 \mathrm{~V}$, is reached at 777 $\mathrm{kHz}$, the peak frequency, for $33 \%$ of water content. The inconsistency on the trend of this data makes it unsuitable for the purpose of this experiment, as seen in Fig. 9.

The data obtained from P3 when testing S1 shows a trend, although the said trend is not suitable for the purpose of this

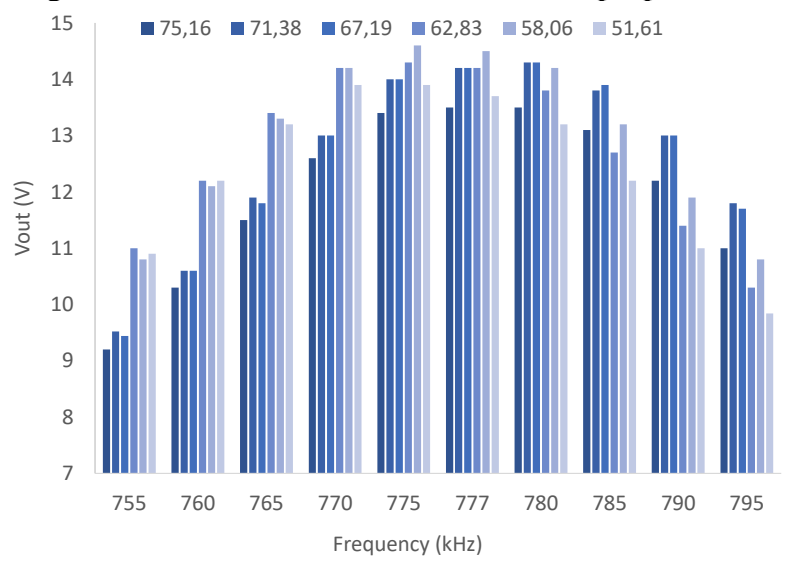

Fig. 8. Vout of P2 using S1

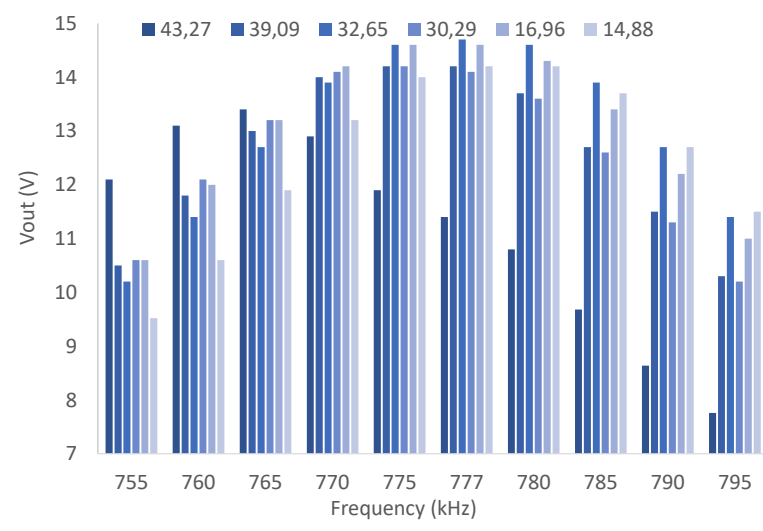

Fig. 9. Vout of P2 using S2 test. At first the Vout increases and suddenly it decreases, see Fig. 10. Since the peak frequency for this prototype is very high, only the lower side was studied. The peak Vout shown in this experiment is $13.9 \mathrm{~V}$. It was observed for the highest frequency tested, $1350 \mathrm{kHz}$, for water content of $67 \%$.

The results from P3 on S2 are completely random. The Vout increases and decreases every other frequency. The peak Vout is $13.7 \mathrm{~V}$, obtained on the frequency $1350 \mathrm{kHz}$ for a $33 \%$ water content. The absence of a trend presented by this prototype makes it unfit for the purpose of this experiment.

\section{Calibration of the equation}

In this subsection, we try to determine whether one sensor can be used for the determination of the soil moisture and in that case, the equation. We present the graphs with the calibrated data. The mathematical model chosen to fit the data is linear regression. This choice was made due to the simplicity of the model.

As proven by the previous graphs, P1 shows good results for S2. Nonetheless, it is useless for S1. The contrary is true for P2. Unfortunately, P3 seems to perform poorly for both soils.

The data for the calibration of P1 with $\mathrm{S} 2$ is the Vout from the tested frequency previous to the peak frequency, $225 \mathrm{kHz}$. We can see a representation of this data along with the calibration equation (Eq. 4) and the $\mathrm{R}^{2}$, see Fig. 12. The $\mathrm{R}^{2}$ is a statistic parameter to estimate the correlation degree between the formula and the data, the higher it is to 1 the more accurate correlation is.

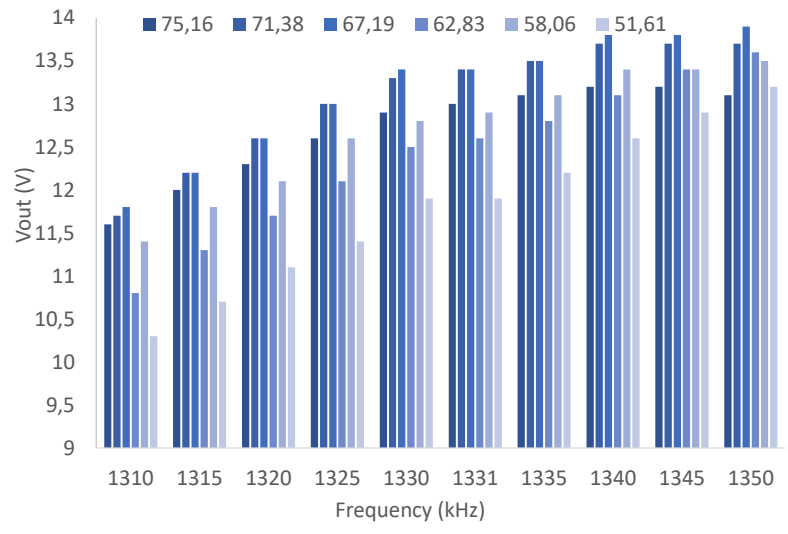

Fig. 10. Vout of P3 using S1

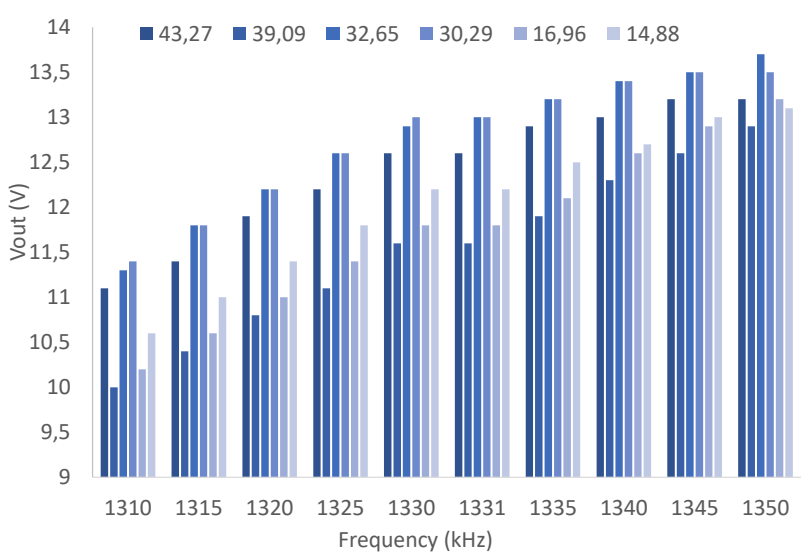

Fig. 11. Vout of P3 using S2 
As expected, due to the use of a prototype with diverse characteristics, the result for P3 with $\mathrm{S} 1$ is different. The data used for this calibration comes from the $1345 \mathrm{kHz}$ frequency and the calibration equation (Eq. 5) along with a representation of this data and the $\mathrm{R}^{2}$ can be seen in Fig. 13.

Conclusion and Future work Precision agriculture has become one of the most important tools in order to better manage resources. Nevertheless, solutions that come from precision agriculture and the use of IoT are often too expensive. Moreover, the low-cost sensors used nowadays must be in direct contact with the soil.

In this paper, we presented three prototypes of sensors, one of which was not in direct contact with the soil. We determined that while none of the sensors could work for both soils, there was a good one for each of the tested soils.

Further tests are needed in order to obtain wider results. The prototypes will be tested in more soils, in order to search for a pattern. Moreover, the prototypes will also be tested under different temperatures to test their sensibility to the changes in the environment.

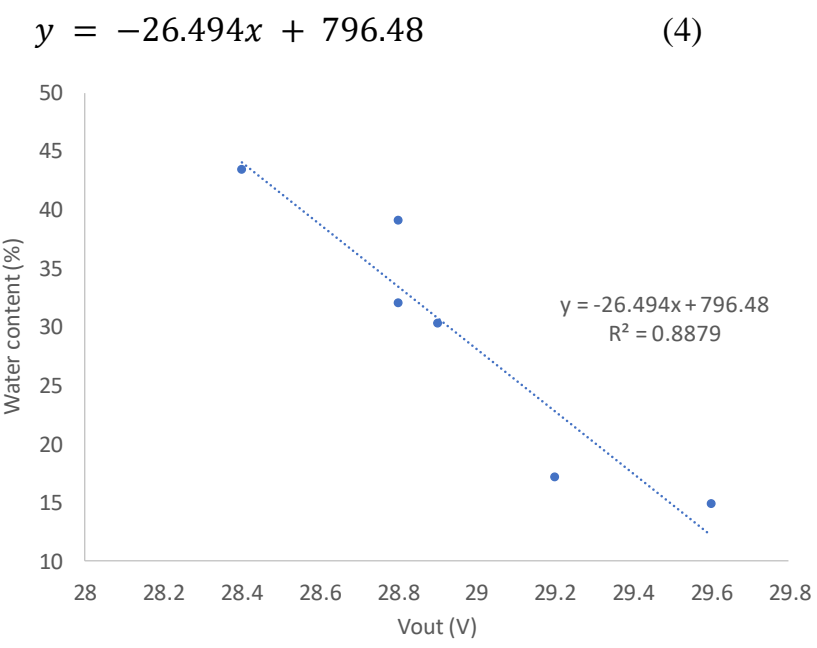

Fig. 12. Vout of P1 with S2 and its mathematical model

$$
y=-8.4075 x+159.66
$$

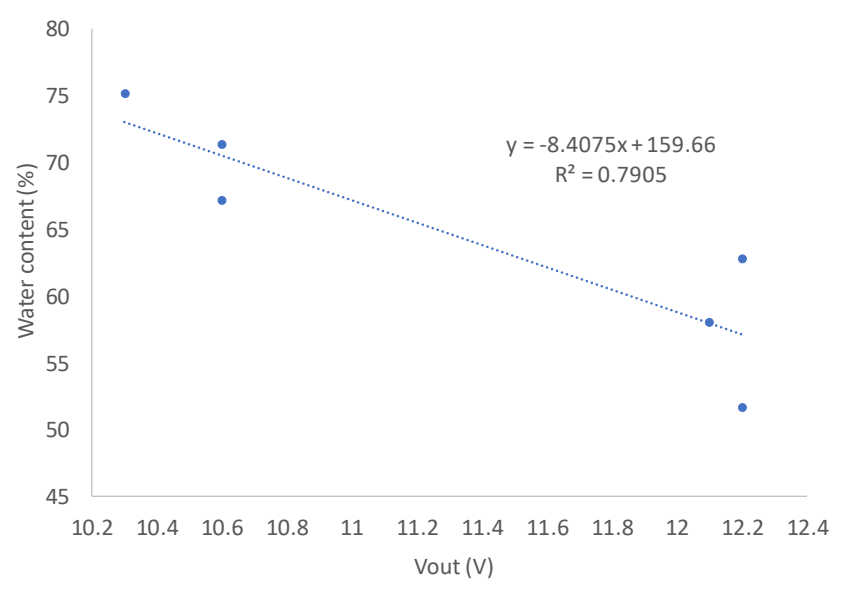

Fig. 13. Vout of P2 with S1 and its mathematical model

\section{ACKNOWLEDGMENT}

This work is partially found by the Conselleria de Educación, Cultura y Deporte with the Subvenciones para la contratación de personal investigador en fase postdoctoral, grant number APOSTD/2019/04, by European Union through the ERANETMED (Euromediterranean Cooperation through ERANET joint activities and beyond) project ERANETMED3-227 SMARTWATIR, and by the European Union with the "Fondo Europeo Agrícola de Desarrollo Rural (FEADER) - Europa invierte en zonas rurales", the MAPAMA, and Comunidad de Madrid with the IMIDRA, under the mark of the PDR-CM 2014-2020" project number PDR18-XEROCESPED.

\section{REFERENCES}

[1] X. Peng, "New multiparametric similarity measure and distance measure for interval neutrosophic set with IoT industry evaluatio," IEEE Access, vol. 7, pp. 28258-28280, 2019.

[2] A. F. Santamaria, P. Raimondo, M. Tropea, F. De Rango, C. Aiello, "An IoT Surveillance System Based on a Decentralised Architecture," Sensors, vol. 19, no. 6, 2019.

[3] A. Veiga, L. Garcia, L. Parra, J. Lloret, V.Augele, “An IoT-based smart pillow for sleep quality monitoring in AAL environments," In Third IEEE International Conference on Fog and Mobile Edge Computing (FMEC), 23-26 April, Barcelona, Spain, pp. 175-180, 2018.

[4] L. Parra, J. Rocher, S. Sendra, J. Lloret, An Energy-Efficient IoT Group-Based Architecture for Smart Cities, In Energy Conservation for IoT Devices, Springer, Singapore, pp. 111-127, 2019.

[5] T. Achouak, B. Khelifa, L. García, L. Parra, J. Lloret, B. Fateh, "Sensor Network Proposal for Greenhouse Automation placed at the South of Algeria," Network Protocols \& Algorithms, vol. 10, no. 4, pp. 53-69, 2018.

[6] M. Parra, L. Parra, J. Rocher, J. Lloret, P. V. Mauri, J. V. Llinares, A Novel Low-Cost Conductivity Based Soil Moisture Sensor. The $2^{\text {nd }}$ International Conference on Advanced Intelligent Systems for Sustainable Development, 8 - 11 July, Maarrakec, Morocco, 2019.

[7] S. U. Susha Lekshmi, D. N. Singh, M. S. Baghini, "A critical review of soil moisture measurement," Measurement, vol. 54, pp. 92-105, 2014.

[8] F. Kizito, C. S. Campbell, G. S. Campbell, D. R. Cobos, B. L. Teare, B. Carter, J. W. Hopmans, "Frequency, electrical conductivity and temperature analysis of a low-cost capacitance soil moisture sensor," Journal of Hydrology, vol. 352, no. 3-4, pp. 367-378, 2008.

[9] L. Gendron, G. Létourneau, L. Anderson, G. Sauvageau, C. Depardieu, E. Paddock, E., ... J. Caron, "Real-time irrigation: Cost-effectiveness and benefits for water use and productivity of strawberries," Scientia horticulturae, vol. 240, pp., 468-477, 2018.

[10] Dursun, M., \& Ozden, S. (2011). A wireless application of drip irrigation automation supported by soil moisture sensors. Scientific Research and Essays, 6(7), 1573-1582.

[11] H. Mittelbach, I. Lehner, S. I. Seneviratne, "Comparison of four soil moisture sensor types under field conditions in Switzerland," Journal of Hydrology, vol. 430, pp. 39-49, 2012.

[12] K. X. Soulis, and S. Elmaloglou, "Optimum soil water content sensors placement for surface drip irrigation scheduling in layered soils," Computers and electronics in agriculture, vol. 152, pp. 1-8, 2018 .

[13] Web page of the current generator used. Available at: https://www.tek.com/arbitrary-function-generator/afg1000-manual. Last access on 09/05/2019

[14] Web page of the utilized oscilloscope. Available at: https://www.tek.com/datasheet/digital-storage-oscilloscopes. Last access on $09 / 05 / 2019$ 